Draft Version November 2, 2011

Preprint typeset using $\mathrm{LAT}_{\mathrm{E}} \mathrm{X}$ style emulateapj v. 11/26/04

\title{
THE SUPERNOVA TRIGGERED FORMATION AND ENRICHMENT OF OUR SOLAR SYSTEM
}

\author{
M. Gritschneder ${ }^{1}$,D.N.C. Lin $^{1,2}$, S.D. MurraY ${ }^{3}$, Q.-Z. Yin ${ }^{4}$, M.-N. Gong ${ }^{5}$ \\ ${ }^{1}$ Kavli Institute for Astronomy and Astrophysics, Peking University, Yi He Yuan Lu 5, Hai Dian, 100871 Beijing, China \\ gritschneder@pku.edu.cn \\ ${ }^{2}$ Astronomy and Astrophysics Department, University of California, Santa Cruz, CA 95064, USA \\ ${ }^{3}$ Lawrence Livermore National Laboratory, University of California, Livermore, CA 94550, USA \\ ${ }^{4}$ Department of Geology, University of California, Davis, CA 95616, USA and \\ ${ }^{5}$ Department of Physics, Tsinghua University, Hai Dian, 100084 Beijing, China \\ Draft version November 2, 2011
}

\begin{abstract}
We investigate the enrichment of the pre-solar cloud core with short lived radionuclides (SLRs), especially ${ }^{26} \mathrm{Al}$. The homogeneity and the surprisingly small spread in the ratio ${ }^{26} \mathrm{Al} /{ }^{27} \mathrm{Al}$ observed in the overwhelming majority of calcium-aluminium-rich inclusions (CAIs) in a vast variety of primitive chondritic meteorites places strong constraints on the formation of the the solar system. Freshly synthesized radioactive ${ }^{26} \mathrm{Al}$ has to be included and well mixed within $20 \mathrm{kyr}$. After discussing various scenarios including X-winds, AGB stars and Wolf-Rayet stars, we come to the conclusion that triggering the collapse of a cold cloud core by a nearby supernova is the most promising scenario. We then narrow down the vast parameter space by considering the pre-explosion survivability of such a clump as well as the cross-section necessary for sufficient enrichment. We employ numerical simulations to address the mixing of the radioactively enriched SN gas with the pre-existing gas and the forced collapse within $20 \mathrm{kyr}$. We show that a cold clump of $10 \mathrm{M}_{\odot}$ at a distance of $5 \mathrm{pc}$ can be sufficiently enriched in ${ }^{26} \mathrm{Al}$ and triggered into collapse fast enough - within $18 \mathrm{kyr}$ after encountering the supernova shock - for a range of different metallicities and progenitor masses, even if the enriched material is assumed to be distributed homogeneously in the entire supernova bubble. In summary, we envision an environment for the birth place of the Solar System 4.567 Gyr ago similar to the situation of the pillars in M16 nowadays, where molecular cloud cores adjacent to an HII region will be hit by a supernova explosion in the future. We show that the triggered collapse and formation of the Solar System as well as the required enrichment with radioactive ${ }^{26} \mathrm{Al}$ are possible in this scenario.

Subject headings: Stars: formation, Stars: protostars, (Stars:) supernovae: general, Hydrodynamics, ISM: kinematics and dynamics, ISM: abundances, Protoplanetary disks, Meteorites
\end{abstract}

\section{INTRODUCTION}

The time-scale for the formation events of our Solar System can be derived from the decay products of radioactive elements found in meteorites. Short lived radionuclides (SLRs) such as ${ }^{26} \mathrm{Al},{ }^{41} \mathrm{Ca},{ }^{53} \mathrm{Mn}$ and ${ }^{60} \mathrm{Fe}$ can be employed as high-precision and high-resolution chronometers due to their short half-lives. These SLRs are found in a wide variety of Solar System materials, including calcium-aluminium-rich inclusions (CAIs) in primitive chondrites (e.g. Lee et al. 1976; Amelin et al. 2002). The decay of ${ }^{26} \mathrm{Al}$ into ${ }^{26} \mathrm{Mg}$ in particular, with a half-life of $\tau \approx 0.7 \mathrm{Myr}$, provides by far the highest resolution measurements that mark the initial formation of the proto-planetary disk (Jacobsen et al. 2008). As ${ }^{26} \mathrm{Al}$ decays, the ratio of ${ }^{26} \mathrm{Al} /{ }^{27} \mathrm{Al}$ at the time of condensation can be directly derived from the present day ${ }^{26} \mathrm{Mg} /{ }^{24} \mathrm{Mg}$ and ${ }^{27} \mathrm{Al} /{ }^{24} \mathrm{Mg}$ measurements. Furthermore, the spread of ${ }^{26} \mathrm{Al} /{ }^{27} \mathrm{Al}$ among different CAIs gives the precise time-span in which these CAIs formed. While there is considerable spread among the CAIs towards lower ratios of ${ }^{26} \mathrm{Al} /{ }^{27} \mathrm{Al}$ due to remelting and thermal metamorphism, the upper value, the so called 'canonical ratio', (MacPherson et al. 1995) is now well established (e.g. Jacobsen et al. 2008; Villeneuve et al. 2009).

The general picture we adopt here is that a certain amount of ${ }^{26} \mathrm{Al}$ is injected in the nascent solar nebula and then gets incorporated into the earliest formed
CAIs as soon as the temperature drops below the condensation temperature of CAI minerals. These CAIs are most frequently found in $\mathrm{CV}$-chondrites ${ }^{1}$. Lower ratios of ${ }^{26} \mathrm{Al} /{ }^{27} \mathrm{Al}$ can then be explained by subsequent episodes of CAI remelting or thermal disturbance, thereby explaining the heterogeneity below the canonical value. Therefore, the CAIs found in chondrites represent the first known solid objects that crystalized within our Solar System and can be used as an anchor point to determine the formation time-scale of our Solar System. Various measurements of different CAIs by several research groups have not only confirmed the canonical ratio of $(5.23 \pm 0.13) \times 10^{-5}$, but also established a very small spread (for a recent review see Villeneuve et al. 2009). This spread corresponds to an age range of less than $\simeq 20 \mathrm{kyr}$ (e.g. Jacobsen et al. 2008).

In addition, the Mg-isotope composition appears to be fairly uniform among bulk chondrites as well as Mars, Moon and Earth. This is a strong indication that initially $\mathrm{Al}-$ and $\mathrm{Mg}$-isotopes are distributed quite homogeneously $( \pm 10 \%)$ in the proto-planetary disk (Thrane et al. 2006; Villeneuve et al. 2009; Boss 2011). The extremely small time-span together with the highly homogeneous mixing of isotopes poses a severe challenge for theoretical models on the formation of our Solar System

\footnotetext{
${ }^{1} \mathrm{CV}$-chondrites are a class of CAI-rich carbonaceous chondrites named after the Vigarano meteorite (Dauphas \& Chaussidon 2011)
} 
Various theoretical scenarios for the formation of the Solar System have been discussed. Shortly after the discovery of SLRs, it was proposed that they were injected by a nearby massive star. This can happen either via a supernova explosion (Cameron \& Truran 1977) or by the strong winds of a Wolf-Rayet star (Arnould et al. 1997). Another possibility would be the in situ enrichment inside the disk by the non-thermal activity of the Sun (Shu et al. 2001). Wasserburg et al. (1995) have proposed an asymptotic giant branch (AGB) star as an possible source of SLRs in the Solar System (see also Trigo-Rodríguez et al. 2009).

Up to now, none of these models have been able to explain the extremely small range of CAI condensation ages (the spread of $\approx 20 \mathrm{kyr}$ ). In this paper, we discuss several possible solutions to this problem. First, we show that most of these scenarios are not compatible with an almost coeval formation of CAIs. We then go on to perform numerical simulations on the promising scenario of a type IIa supernova as the trigger of our Solar System.

\section{PHYSICAL PROCESSES AND FEASIBILITY OF SOLUTIONS}

In this section we discuss the physical processes in different scenarios and their viability. For a detailed discussion of these scenarios, including an assessment of their likelihood see the recent review by Adams (2010).

As mentioned before, the canonical value of ${ }^{26} \mathrm{Al} /{ }^{27} \mathrm{Al}$ is established to be $(5.23 \pm 0.13) \times 10^{-5}$. Assuming an total mass for ${ }^{27} \mathrm{Al}$ of $M_{27} \mathrm{Al}=7.1 \times 10^{-5} \mathrm{M}_{\odot}$ (e.g. Lodders 2003 ) we can convert the observed ratio into a total mass of $M_{26 \mathrm{Al}}^{\text {canonical }} \approx 3.71 \times 10^{-9} \mathrm{M}_{\odot}$ of radioactive ${ }^{26} \mathrm{Al}$, which has to be present in the early Solar System.

\subsection{In situ Enrichment}

One solution to the incorporation of ${ }^{26} \mathrm{Al}$ into CAIs was proposed by Shu et al. (2001). Young stars can undergo the so-called $\mathrm{X}$-wind phase. In this phase the combination of magnetic fields, inflowing material and stellar outflows can form a wind between the proto-star and the proto-planetary disk (Shu et al. 1997).

SRLs are produced in this scenario by solar energetic particle irradiation of dust and gas near the protoSun (Shu et al. 2001) and incorporated into CAIs. This mechanism can explain the ratio of ${ }^{10} \mathrm{Be} /{ }^{9} \mathrm{Be}=8.8 \pm$ $0.6 \times 10^{-4}$ inferred from measurements of ${ }^{10} \mathrm{~B}$, the decay product of ${ }^{10} \mathrm{Be}$ (McKeegan et al. 2000). Another way to explain this ratio was proposed by Desch et al. (2004). They calculate the contribution of ${ }^{10} \mathrm{Be}$ trapped by galactic cosmic rays to the collapsing molecular cloud core and conclude that a large amount, if not all, ${ }^{10} \mathrm{Be}$ can be attributed to cosmic rays.

For the purpose of this study it is sufficient to note that this mechanism can not explain the observed amount of ${ }^{26} \mathrm{Al}$ due to the following reasons. First of all, ${ }^{26} \mathrm{Al}$ and ${ }^{10} \mathrm{Be}$ do not correlate well in CAIs (e.g. Marhas et al. 2002). In addition, SLRs produced by this mechanism are intrinsically expected to be highly heterogeneous. Variations in their relative abundances would reflect the local energetic particle environment and their episodic nature of production. This is in contradiction to the observed homogeneity $( \pm 10 \%)$ of ${ }^{26} \mathrm{Al}$. Furthermore, the amount of ${ }^{26} \mathrm{Al}$ which can be produced by this mechanism is at least an order of magnitude too low to explain the canonical value (Duprat \& Tatischeff 2007, 2008).

\subsection{External Enrichment by AGB stars}

Another source of ${ }^{26} \mathrm{Al}$ are AGB stars. Every star of $\sim 0.8-8 \mathrm{M}_{\odot}$ (Herwig 2005) undergoes this phase when helium fusion in the core is complete and helium shell burning begins. In this phase, the star loses most of its envelope in stellar winds, which could in term enrich the proto-solar cloud(e.g. Wasserburg et al. 1995). This stage is at the end of a stellar life. However, AGB stars are not present in star forming regions and hence an encounter is very unlikely (Kastner \& Myers 1994). In addition, the total amount of ${ }^{26} \mathrm{Al}$ requires a massive AGB-star and the short enrichment-timescale requires a brief AGB-phase.

\subsection{External Enrichment by Wolf-Rayet stars}

An interesting possibility was proposed by Tatischeff et al. (2010) who followed up on an earlier idea by Arnould et al. (1997). Here, a run-away Wolf-Rayet star that gets ejected from its parental cluster of massive stars sweeps up a smaller shell while traveling into the ISM. This shell gets enriched by material from the strong stellar winds of the Wolf-Rayet star. As soon as this star explodes in a supernova it sweeps up an even denser shell which expands rapidly. After $\simeq 10 \mathrm{kyr}$ the shell starts to cool and a cold core can start to form due to dynamical and gravitational instabilities which subsequently undergoes collapse. During this collapse phase the the surface density of the core increases with time (Whitworth et al. 1994; Heitsch et al. 2008). Since this theory assumes that the ${ }^{26} \mathrm{Al} /{ }^{27} \mathrm{Al}$ ratio increases as the surface density of the clump increases, the core becomes enriched sufficiently to explain the observed values in CAIs after $10^{5}-10^{6} \mathrm{yr}$. However, there is no physical reason why this ratio should increase as the core density rises. The initial ratio within the shell should be conserved and only modified by radioactive decay (the decay is taken into account by Tatischeff et al. 2010).

A similar scenario was proposed by Gaidos et al. (2009). Here, an entire molecular cloud $\left(M>10^{5} \mathrm{M}_{\odot}\right)$ is enriched in ${ }^{26} \mathrm{Al}$ by a nearby star forming region. After $\sim 4-5 \mathrm{Myr}$ this cloud begins to form stars, including the Sun. During this stage, the Solar System is enriched with heavier SLRs. This scenario can explain the different abundances of SLRs (see 4 ). However, it requires a precise chain of events. In addition, this theory assumes continuous enrichment which is at odds with the small age spread of CAIs (see below).

\subsection{Constraints on continuous enrichment}

All three scenarios described above are based on the assumption of a continuous enrichment. In that case, the characteristic time scale for the enrichment with radioactive isotopes is much longer than the brief duration $(<20 \mathrm{kyr})$ of the CAI formation episode which is inferred from the meteoritic data.

In external enrichment models, sufficient radioactive isotopes can only be intercepted by a tenuous and extended solar-system progenitor cloud prior to its collapse. But the free fall time scale for such a cloud is generally $\sim 100 \mathrm{kyr}$ or longer. If the central star and disk accrete material from this parent envelope on such a time 
scale, dust and grains would be processed with different rates at different locations and in different stages. During this entire phase, the assembly and formation of the CAIs would be possible, leading to inhomogeneities and an age-spread of $\sim 100 \mathrm{kyr}$.

This is inconsistent with the observationally inferred homogeneity of $\mathrm{Mg}$ - and $\mathrm{Al}$-isotopes during the formation epoch of CAIs. Here, we make a clear distinction and differentiate the initial formation epoch of CAIs from later epochs when some CAIs subsequently experienced a complex thermal history within the solar nebula and metamorphism on their host meteorite parent asteroids extending over 0.1 - 3 Myr (e.g. MacPherson et al. 1995; Hsu et al. 2000; Young et al. 2005; MacPherson et al. 2007). One possible mechanism to homogenize the isotope's distribution and reset the clock for grain condensation and CAI formation is to heat the infalling gas above the condensation temperature for CAIs of $\sim 1500-1800 \mathrm{~K}$ (e.g. Grossman 2002; Lodders 2003). During the infall, accretion and shocks can dissipate sufficient energy and heat up the disk to this temperature close to the central star (within $\sim 0.1 \mathrm{AU}$ ). In order for the infalling gas to arrive at this location and form a compact disk, it must carry very little angular momentum. However, turbulent angular momentum transport, induced either by gravitational or magneto-rotational instabilities, would lead to mass diffusion. When the disk spreads beyond an AU, its mid-plane cools (Ruden \& Lin 1986; Garaud \& Lin 2007) well below the condensation temperature of the CAIs. If the infalling gas has a modest amount of angular momentum, it would form more extended disks with shock temperatures below $10^{3} \mathrm{~K}$ at a distance outside $1 \mathrm{AU}$ (Walch et al. 2009). In either limit, condensation of the elements and the formation of CAI's would continue until infall is terminated.

A possible solution to reconcile with CAI's brief formation epoch is to assume that all infalling gas was heated above their condensation temperature of $\sim 1800 \mathrm{~K}$ and then cooled within $20 \mathrm{kyr}$. Such a scenario would require a very compact disk and therefore a violent, external cause, i.e. a supernova, to trigger and drive the collapse of the entire progenitor cloud which formed the Solar System. As shown in Appendix A, the subsequent growth of CAIs would be possible on a short enough time-scale in such a disk.

\subsection{External Enrichment by a Type II Supernova}

A frequently discussed possibility for SLRs is the external enrichment by a type IIa supernova (SN IIa). Huss et al. (2009) estimate that a supernova with a progenitor of $20-60 \mathrm{M}_{\odot}$ can produce the required SLRs. From the simple assumption that the cross-section of the enriched region has to be high enough to intercept enough ${ }^{26} \mathrm{Al}$ we can derive a size-distance relation from geometrical considerations:

$$
R=\sqrt{\frac{4 \cdot M_{\mathrm{Al}_{\odot}^{26}}}{M_{\mathrm{Al}_{\mathrm{SN}}^{26}}}} D
$$

Where $R$ is the radius of the intercepting cloud or disk and $D$ is the distance from the supernova. $M_{\mathrm{Al}_{\odot}^{26}}$ and $M_{\mathrm{Al}_{\mathrm{SN}}^{26}}$ are the amount of ${ }^{26} \mathrm{Al}$ required in the Solar System and produced in the supernova, respectively. This

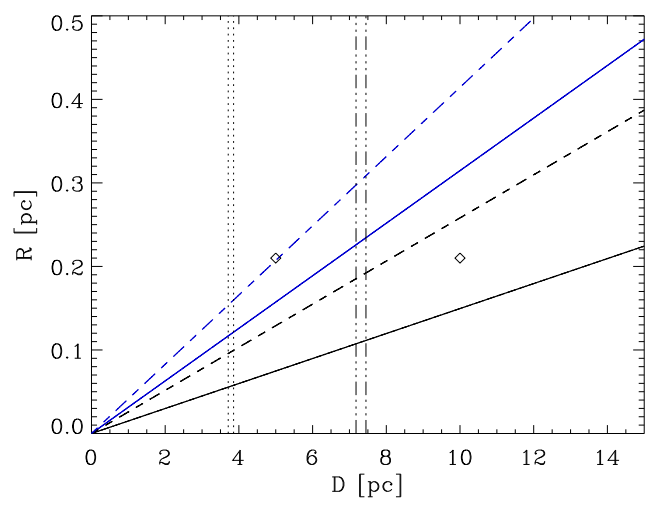

FIG. 1. - The parameter space. Plotted is the distance D from the exploding star versus the radius $\mathrm{R}$ of the pre-solar clump. The diagonal lines give the required ratio for the enrichment. Black is for a $40 \mathrm{M}_{\odot}$ star, blue for a $20 \mathrm{M}_{\odot}$ star, solid is solar metallicity with $\mathrm{Z}=0.02$, dashed is $\mathrm{Z}=0.004$. The vertical lines are the radius of the HII-region for an O5V star after $3 \mathrm{Myr}$ and a B0V star after $10 \mathrm{Myr}$, respectively - dotted is for a cold medium of $5 \times 10^{4} \mathrm{~cm}^{-3}$, dash-dotted for $5 \times 10^{3} \mathrm{~cm}^{-3}$. The diamonds represent the parameters chosen for the simulations in 3

\begin{tabular}{lcc}
\multicolumn{3}{c}{$M_{\mathrm{Al}_{\mathrm{SN}}^{26}}\left[\mathrm{M}_{\odot}\right]$} \\
\hline Mass $\left[\mathrm{M}_{\odot}\right]$ & $\mathrm{Z}=0.02$ & $\mathrm{Z}=0.004$ \\
\hline 20 & $1.5 \times 10^{-5}$ & $8.65 \times 10^{-6}$ \\
40 & $6.64 \times 10^{-5}$ & $2.23 \times 10^{-5}$
\end{tabular}

TABLE 1

TYPICAL ${ }^{26} \mathrm{Al}$ YIELDS IN $\mathrm{M}_{\odot}$ FOR DIFFERENT SUPERNOVA PROGENITOR MASSES AND METALLICITIES TAKEN FROM NOMOTO ET AL. (2006).

equation is of course simplified, e.g. neglecting radioactive decay as the time-scales are very short, as well as assuming a perfect mixing efficiency, i.e. all ${ }^{26} \mathrm{Al}$ intercepted is assumed to be mixed into the Solar System.

\subsubsection{Enrichment in the Disk Stage}

From the values in Table 1 one can calculate that at solar metallicity a $40 \mathrm{M}_{\odot}$ progenitor supernova could deposit enough ${ }^{26} \mathrm{Al}$ in a $1000 \mathrm{AU}$ face-on disk at $D=$ $0.3 \mathrm{pc}$ or a $20 \mathrm{M}_{\odot}$ progenitor enough ${ }^{26} \mathrm{Al}$ at $D=0.15 \mathrm{pc}$. That is very close to the hot, ionizing star. Although a proto-stellar disk could survive in this violent environment (Adams 2010), there are additional timing issues. For example Gounelle \& Meibom (2008) estimate the probability that the proto-solar system is young enough $(<1 \mathrm{Myr})$, able to survive photo-evaporation and close enough to get sufficiently enriched $(D<0.3 \mathrm{pc})$ to be less than $10^{-3}$ in the most favorable case. Based on the required young age of the system, Williams \& Gaidos (2007) argue for a very massive supernova progenitor, which in turn leads to a short life-time, i.e. an early supernova. As very massive stars are only found in massive clusters, they conclude that the likelihood is $<1 \%$.

Ouellette et al. (2005, 2009, 2010) suggested an intriguing possibility where the disk is further away from the supernova $(\sim 2 \mathrm{pc})$ and the enrichment is achieved via the injection of already formed grains into the presolar disk ('aerogel' model). To achieve the high observed yield, the supernova gas has to be highly clumpy and the clumps have to be highly enriched in SLRs - 
to this possibility the authors estimate a probability of $\sim 10^{-3}-10^{-2}$. There are, however, additional uncertainties. Recent models (e.g. Ida \& Lin 2004) suggest that the minimum mass solar nebula might be more massive than their assumed $0.01 \mathrm{M}_{\odot}$, which would require an even more efficient enrichment. Future investigation on the clumpiness of SN-shock fronts are desirable (see 44). A remaining issue in this picture is the question why this scenario does not result in multiple enrichments of the early Solar System. After the first enrichment by a supernova, which leads to the canonical value of ${ }^{26} \mathrm{Al} /{ }^{27} \mathrm{Al}$, it is likely that several more lighter and therefore more long-lived stars in the cluster undergo subsequent supernova. This would result in several enrichment episodes during the entire lifetime of the disk ( $>10 \mathrm{Myr})$.

\subsubsection{Enrichment in the Cloud Core Stage}

The enrichment of the pre-solar cloud core by a supernova has been suggested for a long time (Cameron \& Truran 1977; Cameron et al. 1995). Recently, Boss et al. (2008, 2010) and Boss \& Keiser (2010) investigated the implosion and enrichment of a $1 \mathrm{M}_{\odot}$ cloud core in detail. They assume the cloud core to be more than $10 \mathrm{pc}$ away from the progenitor. Therefore, the supernova front has slowed down considerably by the time it reached the pre-solar cloud. In addition, it has a finite thickness. In their models, the shock speed ranges from $v_{\mathrm{S}}=1-100 \mathrm{~km} \mathrm{~s}^{-1}$ (Boss et al. 2010), the shock thickness from $\delta_{\mathrm{S}}=3 \times 10^{-4}-3 \times 10^{-2} \mathrm{pc}$ and the shock density from $\rho_{\mathrm{S}}=3.6 \times 10^{-21}-2.4 \times$ $10^{-17} \mathrm{~g} \mathrm{~cm}^{-3}$ (Boss \& Keiser 2010). They find that with certain initial conditions the enrichment is possible in their model. For example a thin $\left(\delta_{\mathrm{S}}=3 \times 10^{-4}\right)$, dense ( $\left.\rho_{\mathrm{S}}=6 \times 10^{-18} \mathrm{~g} \mathrm{~cm}^{-3}\right)$ shock at a rather low velocity $\left(v_{\mathrm{S}}=20 \mathrm{kms}^{-1}\right)$ and therefore far away from the source can enrich the clump sufficiently. This can be easily understood as they assume that the enriched supernova material is completely within the shock. Therefore, as the geometrical dilution rises, the shock has to be thinner to contain a sufficient amount of supernova material. In addition, the time-scale of the collapse is longer $(t>40 \mathrm{kyr})$ than the value inferred from CAIs in this scenario. Again, this can be attributed to the slower supernova shock further away from the progenitor. Nevertheless, this is an encouraging development and we think this scenario is the most promising.

Bearing the geometrical dilution in mind (cf Fig. 1) we revisit this scenario but place the pre-solar clump closer to the supernova. Therefore, the shock velocities are much higher (see 3.2.1). We also take the more conservative assumption that the material is spread out in the hot phase or at least in a ring at the border within the supernova explosion bubble (see 3.2 .2 and 3.2.3). Furthermore, we do not assume the shock already formed a density enhancement. Instead, we setup the supernova blast wave in the velocities under the assumption of a thin shock. As the evolution is expected to be much more violent, we investigate the collapse of a heavier $\left(10 \mathrm{M}_{\odot}\right)$ cloud core (see \$3.2.1). To assess the feasibility of this initial condition, we address the aspect of survival of the pre-solar cloud (3.1).

A slightly different approach would be the enrichment in the cloud stage without triggering the collapse. Then, the cold cloud would collapse in an 'isolated' manner at a later stage. However, there are clear short-comings of this scenario. To allow for the tight spread of only $\simeq 20 \mathrm{kyr}$, the collapse would have to lead to a stage, where the entire disk is heated above $\sim 1800 \mathrm{~K}$ and then homogeneously cools to lower temperatures within $20 \mathrm{kyr}$ (see also 92.4). To achieve the required heating from the gravitational energy the accretion disk would have to be $<0.5 \mathrm{AU}$. At this scale, the disk could then cool coherently, the CAI could condense and later migrate outwards to explain the homogeneous ${ }^{26} \mathrm{Al} /{ }^{27} \mathrm{Al}$ in CAIs in different meteorites.

However, isolated collapse leads to larger, cooler disks. Thus, a triggering event will be required to force the collapse to a small scale. It is straightforward to assume that this triggering event was the same event as the enrichment, i.e. the supernova shock. Thus, we investigate the triggered case in more detail.

\section{MODEL AND SIMULATIONS}

In this section, we address the parameter space by accessing the enrichment, survivability and existence of the pre-solar cloud core in or adjacent to the HII region of the massive star. We investigate the mixing and the collapse probabilities by employing numerical simulations.

In Fig. 11we plot the distance of the clump to the progenitor star versus the size of the clump. From the geometrical enrichment cross-section discussed before, we can derive a first constraint. Taking the yields in Table 1 into account we plot Eq. 1]. These diagonal lines denote the maximum enrichment efficiency. Even if all the material intersected by the initial cloud ends up in the Solar System (100\% efficiency), the initial condition has to be above these lines to explain the ratio inferred from CAIs.

\subsection{Survival}

The most obvious proof for the existence of cold cloud cores in the proximity of massive star comes from observations. In the pillars in M16 (e.g. Hester et al. 1996) McCaughrean \& Andersen (2002) find several cold cores in almost every region of the pillars. These pillars are about 2 pc away from the ionizing sources, the age of the region is estimated to be $1-2 \mathrm{Myr}$ (Hester et al. 1996). This agrees well with our assumption of structures being present at $5 \mathrm{pc}$ after $3 \mathrm{Myr}$ (see below).

From a theoretical point of view, recent simulations on the formation and evolution of HII-regions show the ubiquity of these structures (e.g. Mellema et al. 2006; Krumholz et al. 2007; Gritschneder et al. 2009b; Arthur et al. 2011). These simulations focus mostly on the ionization of turbulent molecular clouds. As shown in Gritschneder et al. (2010), the average time evolution of the front position is still well approximated by an analytic solution as given in Shu (1991):

$$
R(t)=R_{\mathrm{s}}\left(1+\frac{7}{4} \frac{c_{\mathrm{s}, \mathrm{hot}}}{R_{\mathrm{s}}}\left(t-t_{0}\right)\right)^{\frac{4}{7}}
$$

where $R_{\mathrm{S}}$ is the initial Stroemgren radius ${ }^{2}$ and $c_{\mathrm{S}, \text { hot }}$ is the sound speed of the hot, ionized gas. Using this equa-

\footnotetext{
2 The Stroemgren radius is the radius which can be immediately ionized by an ionizing source, without taking any hydrodynamic evolution into account (Strömgren 1939).
} 
tion, we can estimate the size of the HII-region. To investigate the most extreme cases, we assume a short-lived $40 \mathrm{M}_{\odot}$-star with a very short life-time of $3 \mathrm{Myr}$ as well as a $20 \mathrm{M}_{\odot}$-star with a life-time of $10 \mathrm{Myr}$ (e.g. Hurley et al. 2000) in different density regions. The resulting radii are depicted by the vertical lines in Fig. 1. Surviving clumps should be to the right of theses lines. Note that we assume a relatively high density in the neutral medium before the ignition of the star in order to reflect the birth environment of massive stars. Mvers (2009) suggest that the birth of stellar groups is associated with hubs of column densities greater than $10^{22} \mathrm{~cm}^{-2}$, for example in Taurus (Goldsmith et al. 2008), which corresponds to the assumed densities here. In addition, the high densities have to be present only in the direct surrounding of the massive stars, as the further evolution given in Eq. 2 only depends on the initial Stroemgren radius (and the sound speed in the hot gas). Altogether, these values are chosen to reflect our current understanding of massive star formation. In addition, Fig. 1 gives the averaged front position, the pillars in the observations as well as the simulations can be a few pc further inside, pertruding into the ionized bubble.

\subsection{Collapse Timescale and Mixing in the Gas Phase}

The remaining issues are whether the gas from the supernova can be efficiently mixed with the gas from the pre-existing core, whether collapse occurs and if so, whether it is fast enough. To test these questions, we employ numerical simulations.

\subsubsection{Initial Conditions}

Because the main focus lies on mixing as well as cooling, we employ the numerical code COSMOS. The nonrelativistic hydrodynamics of COSMOS are discussed in (Anninos et al. 2003). For the models described below, the code is run with the default hydrodynamics solver, which is a total variation diminishing (TVD) approximate Riemann solver and a third-order Runge-Kutta time-marching scheme (Shu \& Osher 1988). Because of the importance of cooling, the code is run in internal energy mode. Shocks are handled with a zone-centered scalar artificial viscosity. Optically thin heating and cooling are included, using equilibrium cooling curves (Dalgarno \& McCray 1972; Boehringer \& Hensler 1989). Cooling by metals assumes solar metallicity. Mixing is followed by the use of a passive tracer, whose value is initialized to be proportional to the gas density behind the blast-wave. The problems are run in two dimensions. Cylindrical symmetry is imposed by taking advantage of the relativistic capability of COSMOS, and running the problems with a cylindrical metric.

The problem region runs from -5 to 10 cloud radii in the direction of the shock, and from 0 to 4 cloud radii in the transverse direction. The large radial size is adopted so as to prevent any boundary affects from altering the cloud evolution. Due to the motion of the cloud after shock passage, we use a uniform grid spacing in the direction of the incoming shock, with 4096 zones along the axis, or 274 zones across the cloud radius. In order to enhance resolution of the collapsed cloud, geometrically increasing zone spacing is used in the radial direction. A total of 512 zones are included radially, with a ratio of 1.005 in their spacing, giving a minimum zone size of 0.0017 times the cloud radius.

We set up an molecular cloud core in isolation, which is going to be hit by the supernova shock-wave. We approximate the core by an marginally stable Bonnor-EbertSphere (Bonnor 1956), the solution of the Lane-Emden equation for hydrostatic equilibrium

$$
\frac{1}{r^{2}} \frac{d}{d r}\left(\frac{r^{2}}{\rho} \frac{d p}{d r}\right)=-4 \pi G \rho,
$$

where gravity and hydrodynamical pressure balance each other. We assume an initial temperature of $T_{\text {cold }}=20 \mathrm{~K}$ and a central number density of $n_{\mathrm{c}}=5.7 \times 10^{4} \mathrm{~cm}^{-3}$, which gives a cloud core of mass $M=10 \mathrm{M}_{\odot}$ with a radius $R_{0}=0.21 \mathrm{pc}$. The sphere is embedded in a warm gas in pressure equilibrium with the sphere at a temperature $T_{\text {warm }}=792 \mathrm{~K}$. This is realistic, as the core is embedded in the interstellar medium close to an HII-region. As this is an idealized case and the main focus of this study is the enrichment with SRLs, we don't take any rotational or turbulent motion (e.g. Walch et al. 2010) inside the cloud core into account. While this initial rotation might be important in the later phases of the collapse - especially in determining the final disk size - its influence will be very small in the initial phase here, as the rotational velocities are orders of magnitude smaller than the shock speeds. In addition, the total angular momentum carried by a minimum mass nebula $\left(0.02 \mathrm{M}_{\odot}\right)$ out to $30 \mathrm{AU}$ corresponds to that of $1 \mathrm{M}_{\odot}$ of material at $0.6 \mathrm{AU}$. Unless there is a substantial angular momentum loss since the initial collapse to the setup of a minimum mass nebula, the initial cloud must carry very little angular momentum. In such a low-angular momentum cloud, rotation is unlikely to inhibit the collapse until the size of the cloud is well below the resolution of our simulations and the dynamical time scale is much shorter than the $20 \mathrm{kyr}$ of interest.

The supernova shock-wave at the distances considered here $(5-10 \mathrm{pc})$ has not yet reached the snow-plow phase. So the shock is still in the energy dominated phase and can be approximated by a Sedov-Taylor blast wave (Taylor 1950; Sedov 1959). Following Woosley \& Weaver (1995) we assume an energy release of $E=10^{51} \mathrm{erg}$ for core-collapse supernovae with progenitor stars in the range of $20-40 \mathrm{M}_{\odot}$. Considering the parameter space (3.1), we perform two simulations, placing the cloud core at a distance $D=5 \mathrm{pc}$ (case I) and $D=10 \mathrm{pc}$ (case II), respectively, from the supernova. We evaluate the Sedov solution for the given distances. In case I, the results are that the blast wave reaches the core after $\simeq 8.5 \mathrm{kyr}$ with a post-shock speed of $v_{\mathrm{s}}=276 \mathrm{~km} \mathrm{~s}^{-1}$. The soundspeed in the post-shock gas is $c_{\mathrm{s}}=129 \mathrm{~km} \mathrm{~s}^{-1}$. In case II the cold cloud core is reached after $\simeq 48.2 \mathrm{kyr}$ with $v_{\mathrm{s}}=97.5 \mathrm{~km} \mathrm{~s}^{-1}$ and $c_{\mathrm{S}}=45.4 \mathrm{~km} \mathrm{~s}^{-1}$.

\subsubsection{Case $I$}

In the first case, we place the cold pre-solar core at a distance $D=5 \mathrm{pc}$ from the supernova. Fig. 2 shows the time evolution of the density in this simulation. The shock wave is propagating from the bottom to the top. As it can be clearly seen, the shock wave encompasses the cold core rapidly. Various hydrodynamical instabilities occur at the interface. After $t=4.15 \mathrm{kyr}$ the 

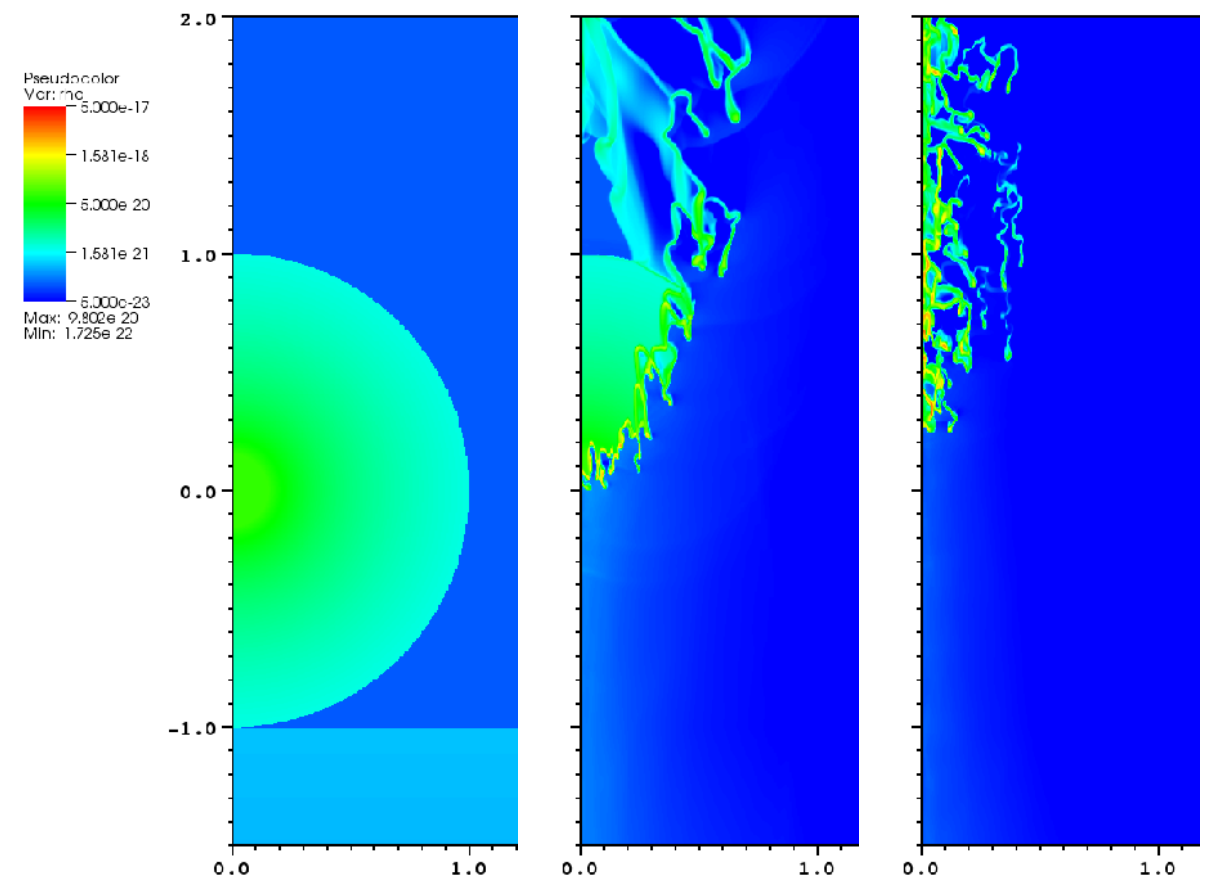

Fig. 2. - The time evolution of case I. Color coded is the density at $t=0 \mathrm{kyr}, t=4.16 \mathrm{kyr}$ and $t=8.33 \mathrm{kyr}$. The length scale is given in units of the radius of the initial cold core $\left(R_{0}=0.21 \mathrm{pc}\right)$.

front has already passed the core. The part of the front which hit the clump continues to interact with the preexisting core, leading to two fronts wrapping around it. As these fronts collide, the material gets mixed with the pre-existing cold gas and is driven into collapse. After $t=8.33 \mathrm{kyr}$ the central region is already at a very high density $\left(\rho_{\max }=3 \times 10^{-15} \mathrm{~g} \mathrm{~cm}^{-3}\right)$, the computation gets expensive and we terminate it.

Fig. 3 shows a zoom-in into the density at the final snapshot. In Fig. 4 we show the temperature distribution in this region. As one can already see, the central region is cold. We define the central region as the cold, dense material between 0.25 and 0.75 on the yaxis of Fig. 4. corresponding to an elongated filament of $\approx 0.1 \mathrm{pc}$ diameter. The mass in this region below a temperature of $20 \mathrm{~K}$ is $M_{\text {core }} \simeq 0.13 \mathrm{M}_{\odot}$, the average density is $\bar{\rho}=1 \times 10^{-17} \mathrm{~g} \mathrm{~cm}^{-3}$. This gives a Jeans-mass ${ }^{3}$ of $\simeq 0.08 \mathrm{M}_{\odot}$. The core is twice as heavy and therefore will definitely undergo collapse. This collapse will proceed at least with the free-fall time-scale, which is $t_{\mathrm{ff}} \approx 20 \mathrm{kyr}$ at this average density. More likely, it will proceed faster as the central density is two orders of magnitude higher and since the material is already moving inwards. In summary, it is probably safe to say that the further collapse to a stage below $1800 \mathrm{~K}$, where the CAIs form, takes less than $10 \mathrm{kyr}$.

The mass is still lower than the solar value, but given the fact that cooling and accretion will still happen this value can increase. Although, given from the mass available in the surrounding we do not estimate it to reach more than $0.2 \mathrm{M}_{\odot}$ in total. In addition, very strong inflows or colliding remnants of the shock are still present,

3 The Jeans-mass is calculated with a mean molecular weight of $\mu=2.5$, i.e. assuming that molecular hydrogen and helium are the dominant species. flowing towards the centre with velocities of $\simeq 10 \mathrm{~km} \mathrm{~s}^{-1}$. These will lead to an enhanced, fast collapse likely force the material in a small central region, which favors the simultaneous crystallization of CAIs.

The other main focus here is the mixing of the enriched hot supernova gas with the cold pre-existing clump. To follow this evolution, we include a tracer in the postshock gas inside the shock-front. The ratio of ${ }^{26} \mathrm{Al}$ to the total gas mass within the supernova bubble depends on several factors. First of all, different progenitor masses give different yields. Secondly, the exact distribution of the enriched gas within the supernova bubble remains an open question.

We investigate two cases here. The worst case, where the supernova enrichment is fully mixed with the entire material in the hot bubble, i.e. the $20 \mathrm{M}_{\odot}$ of total supernova yield are diluted with $\sim 1000 \mathrm{M}_{\odot}$ within the bubble $^{4}$. The second case is more optimistic, assuming that the enriched material stays close to the blast-wave. We assume it to be within the outer $10 \%$ of the radius of the bubble, leading to a dilution by only $\sim 350 \mathrm{M}_{\odot}$ of pre-supernova material.

However, even in the worst case of complete mixing, the enrichment is sufficient for a $40 \mathrm{M}_{\odot}$ progenitor star at solar metallicity. This is shown in Fig. 5. Color coded is the ratio of ${ }^{26} \mathrm{Al}$ to total mass, normalized to the required value of $\simeq 3.71 \times 10^{-9}$. Since in the collapsing regions the ratio is of order unity or higher, we conclude that the gas phase is sufficiently enriched to allow for the formation of the observed CAIs later on.

In Table 2 we list the outcome of different scenarios. We give $M_{26}^{\operatorname{sim}}$, the total mass of ${ }^{26} \mathrm{Al}$ inside the final

4 To estimate the dilution we assume an average density of $n=$ $100 \mathrm{~cm}^{-3}$ within the supernova bubble from here on. This is a high value, but still realistic (for a discussion see 44). 


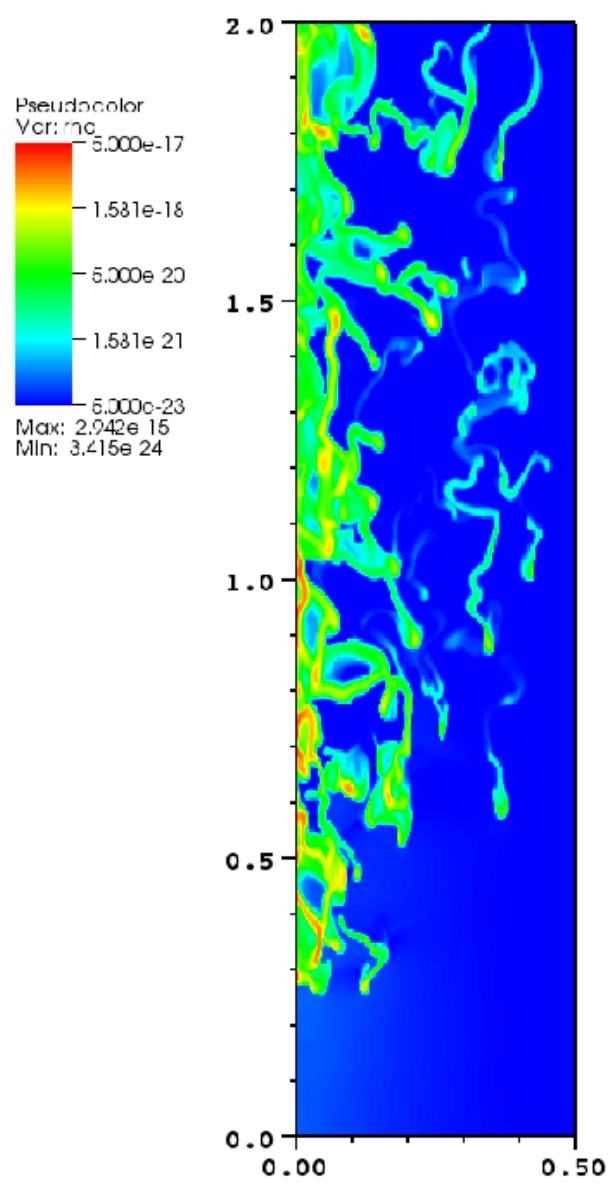

Fig. 3.- Zoom in into the final stage of Case I at $t=8.33 \mathrm{kyr}$. Color coded is the density, the contours denote the gravitational potential. The length scale is given in units of the radius of the initial cold core $\left(R_{0}=0.21 \mathrm{pc}\right)$. As can be clearly seen the densest regions will undergo gravitational collapse.

collapsing core, as well as the dilution $f_{0}$ (i.e. the fraction of supernova material injected into the final core) and the injection efficiency $f_{\mathrm{i}}$ as defined in Eq. 6. Finally, we list the resulting enrichment for a collapsing core scaled to the solar ratio

$$
f_{\odot}=\frac{M_{26 \mathrm{Al}}^{\text {sim }}}{M_{\text {core }}} \frac{M_{\odot}}{M_{26 \mathrm{Al}}^{\text {canonical }}} .
$$

$f_{\odot}$ can be interpreted as the success of the model, i.e. value of 1.0 corresponds to the observed ${ }^{26} \mathrm{Al} /{ }^{27} \mathrm{Al}$ in the Solar System.

Our results differ depending on the assumed distribution of enriched material inside the supernova bubble and on the progenitor mass. As can be seen, in several cases the enrichment is sufficient, if the cloud core is placed at $D=5 \mathrm{pc}$ from the supernova initially. Note that the dilution $f_{0}$ is systematically lower than the values of $f_{0} \approx(0.5-6) \times 10^{-4}$ as given by Takigawa et al. $(2008)$. At the same time the mass in the cold core is only $0.13 \mathrm{M}_{\odot}$ in our case, explaining that difference of one order in magnitude. In addition, we assume a high number density inside the supernova bubble $\left(n=100 \mathrm{~cm}^{-3}\right)$, which leads to a very strong dilution. Thus, these estimates are very conservative and can be viewed as a worst case scenario enrichment.

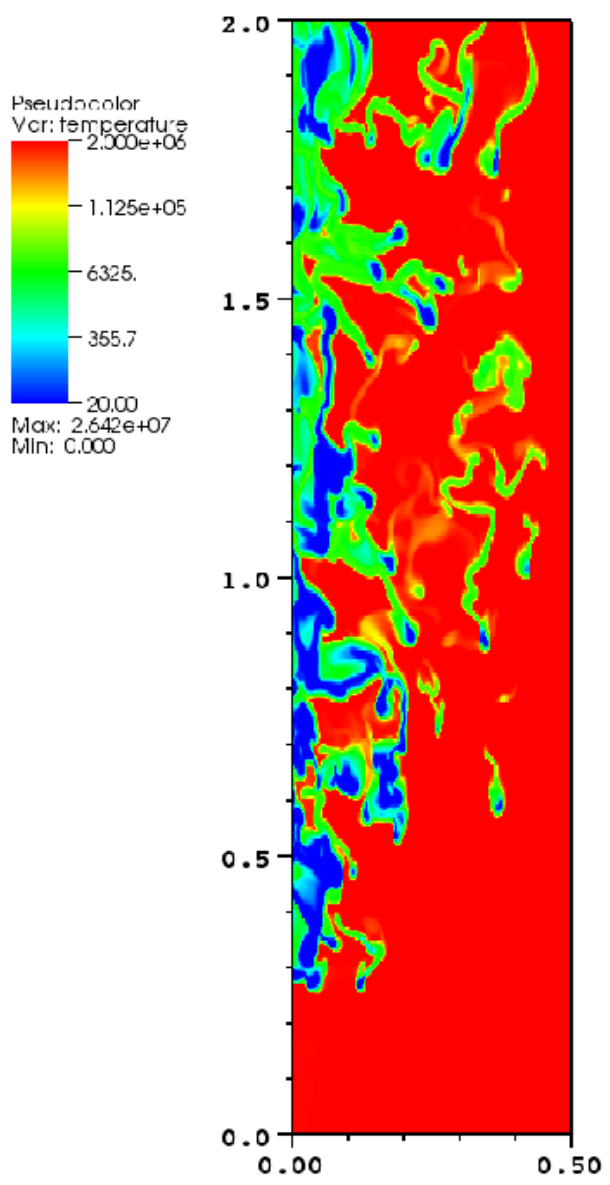

FIG. 4. - The temperature distribution at the final stage at $t=$ $8.33 \mathrm{kyr}$. The blue are cold regions, red are hot regions. The length scale is given in units of the radius of the initial cold core $\left(R_{0}=\right.$ $0.21 \mathrm{pc}$ ). The dense regions are very cold, indicating gravitational collapse.

To further understand the reason for this very efficient mixing, it is useful to take a closer look at the earlier stage of the simulation. In Fig. 6 we show the temperature distribution at the intermediate stage $(t=1.25 \mathrm{kyr})$. As can be clearly seen the material is already cooling behind the shock front. This cold, enriched clumps are mixing very efficiently with the pre-existing core. Therefore, a precise prescription of cooling processes is vital to investigate enrichment scenarios.

\subsubsection{Case II}

In the second case, the cold cloud core is at a distance $D=10 \mathrm{pc}$ and is embedded in an ambient medium which is a factor of ten less dense with a ten times higher temperature than case I. Therefore, the ambient pressure is the same. Due to the longer distance the angular crosssection is smaller, the enriched material is already more dispersed. In addition, the shock has already lost more energy, i.e. the front speed is slower.

The effect of this can be seen in Fig. 7. After $t=$ $14.6 \mathrm{kyr}$ the cold core is still much less deformed by the supernova and not driven into collapse. Although the mixing seems to be very efficient, a closer look at Fig. 8 shows that the gravitational collapsing region, a remnant 


\begin{tabular}{|c|c|c|c|c|c|c|c|}
\hline Distance & Mass & Metallicity & SNe mixing & $M_{26 A}^{\operatorname{sim}}\left[\mathrm{M}_{\odot}\right]$ & $f_{0}$ & $f_{\mathrm{i}}$ & $f \odot$ \\
\hline \multirow[t]{8}{*}{$5 \mathrm{pc}$} & $40 \mathrm{M}_{\odot}$ & $Z=0.02$ & shell & $1.6 \times 10^{-9}$ & $2.3 \times 10^{-5}$ & $5.3 \times 10^{-2}$ & 3.34 \\
\hline & & & complete & $4.4 \times 10^{-10}$ & $6.6 \times 10^{-6}$ & $1.5 \times 10^{-2}$ & 0.91 \\
\hline & & $Z=0.004$ & shell & $5.4 \times 10^{-10}$ & $2.3 \times 10^{-5}$ & $5.3 \times 10^{-2}$ & 1.1 \\
\hline & & & complete & $1.6 \times 10^{-10}$ & $7.0 \times 10^{-6}$ & $1.6 \times 10^{-2}$ & 0.32 \\
\hline & $20 \mathrm{M}_{\odot}$ & $Z=0.02$ & shell & $3.6 \times 10^{-10}$ & $2.5 \times 10^{-5}$ & $5.6 \times 10^{-2}$ & 0.75 \\
\hline & & & complete & $1.0 \times 10^{-10}$ & $6.6 \times 10^{-6}$ & $1.5 \times 10^{-2}$ & 0.21 \\
\hline & & $Z=0.004$ & shell & $1.6 \times 10^{-10}$ & $2.5 \times 10^{-5}$ & $5.6 \times 10^{-2}$ & 0.43 \\
\hline & & & complete & $5.7 \times 10^{-11}$ & $6.5 \times 10^{-6}$ & $1.5 \times 10^{-2}$ & 0.11 \\
\hline \multirow[t]{8}{*}{$10 \mathrm{pc}$} & $40 \mathrm{M}_{\odot}$ & $Z=0.02$ & shell & $3.9 \times 10^{-12}$ & $5.8 \times 10^{-8}$ & $5.2 \times 10^{-4}$ & 0.065 \\
\hline & & & complete & $1.1 \times 10^{-12}$ & $1.6 \times 10^{-8}$ & $1.5 \times 10^{-4}$ & 0.017 \\
\hline & & $Z=0.004$ & shell & $1.3 \times 10^{-12}$ & $5.8 \times 10^{-8}$ & $5.2 \times 10^{-4}$ & 0.022 \\
\hline & & & complete & $3.8 \times 10^{-13}$ & $1.8 \times 10^{-8}$ & $1.6 \times 10^{-4}$ & 0.0064 \\
\hline & $20 \mathrm{M}_{\odot}$ & $Z=0.02$ & shell & $8.7 \times 10^{-13}$ & $5.8 \times 10^{-8}$ & $5.2 \times 10^{-4}$ & 0.014 \\
\hline & & & complete & $2.4 \times 10^{-13}$ & $1.6 \times 10^{-8}$ & $1.5 \times 10^{-4}$ & 0.0041 \\
\hline & & $Z=0.004$ & shell & $5.1 \times 10^{-13}$ & $5.9 \times 10^{-8}$ & $5.4 \times 10^{-4}$ & 0.0085 \\
\hline & & & complete & $1.4 \times 10^{-13}$ & $1.6 \times 10^{-8}$ & $1.4 \times 10^{-4}$ & 0.0023 \\
\hline
\end{tabular}

TABLE 2

Outcome of different scenarios. We give the Distance, mass, Chosen metallicity and assumed SNe mixing. The corresponding yields are taken from Nomoto et AL. (2006). We give $M_{26}^{\text {sim }}$, the total mass of 26 Al inside the final core, as

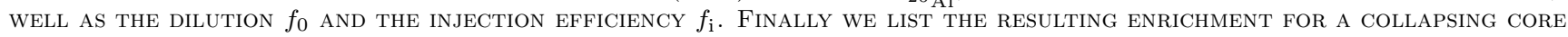
SCALED UP TO THE SOLAR MASS, I.E. A VALUE OF 1.0 OR BIGGER STANDS FOR SUCCESSFUl ENRICHMENT TO THE SOLAR VALUE.

of the original cloud core is not strongly enriched (the blue region at $0.0-0.1$ cloud radii showing the highest density in Fig 7), whereas the enriched region further away (the light blue region between $0.7-1.3$ cloud radii) shows the effect of the two flanks of enriched supernova material colliding.

In addition, the mass in the cold region is much smaller, only $0.01 \mathrm{M}_{\odot}$, which is 30 times less than the Jeans mass at the average density of $\bar{\rho}=7 \times 10^{-19} \mathrm{~g} \mathrm{~cm}^{-3}$ in this region. Therefore, the cloud will not collapse in this case. This is due to supernova shock wave already being substantially weakened so far away from the front. Of course it would be possible to start with a more condensed core, but then the enrichment, which is already difficult in this case (see below), becomes even more unlikely as the radius of the core would be smaller (cf Eq. 1).

From Table 2 it can be seen that sufficient enrichment is very unlikely in this scenario, especially since the enriched material is now more diluted, as the volume of the supernova bubble is bigger, i.e. the material produced by the supernova is now mixed with $\sim 10^{4} \mathrm{M}_{\odot}$ in the complete mixing case and with $\sim 2800 \mathrm{M}_{\odot}$ in the shell case.

Altogether the clump will neither collapse, nor be sufficiently enriched in this case.

\subsection{Comparison of the enrichment in simulations with theoretical (geometrical) predictions}

Here, we compare the predicted enrichment from the geometrical cross-section (Eq. 11 and Fig. 1) with the finally achieved enrichment.

From a geometrical point of view, the dilution $f_{0}$ is given as by the radius of the initial sphere $R_{0}$ and the distance $D$ to the source as:

$$
f_{0, \text { geom }}=\frac{R_{0}^{2} \pi}{4 D^{2} \pi}
$$

Thus, $f_{0, \text { geom }}$ is the amount of ${ }^{26} \mathrm{Al}$ intercepted geometrically by the initial Bonnor Ebert sphere. $f_{0, \text { geom }}=$ $4.4 \times 10^{-4}$ and $f_{0, \text { geom }}=1.1 \times 10^{-4}$ for cases I and II, respectively. The values are systematically higher than $f_{0}$ in Table 2 as Eq. 5 assumes perfect mixing of the supernova material with the background gas. However, it can be used to determine an injection efficiency, which we can not define directly, as we do not assume the material to be in the front initially.

Instead, we define the injection efficiency $f_{\mathrm{i}}$ as the ratio of the finally incorporated mass to expected intercepted mass from geometrical considerations

$$
f_{\mathrm{i}}=\frac{M_{26}^{\mathrm{sim}} \mathrm{Al}}{f_{0, \text { geom }} M_{26 \mathrm{Al}}^{\mathrm{SNe}}}=\frac{f_{0}}{f_{0, \text { geom }}} .
$$

$f_{\mathrm{i}}$ would be unity if all intercepted material is included in the collapsing core. We give the values in Table 2 as well. For case $\mathrm{I} f_{\mathrm{i}} \approx 0.01-0.05$, i.e. a few percent of the material crossing the cold sphere initially are incorporated in the collapsing core finally. In case II, the values are much lower, $f_{\mathrm{i}} \approx 0.0001-0.0006$. First, this is due to a larger amount of un-enriched material in the bigger bubble. Second, the shock front is much weaker in case II, and therefore the front does not mix as efficient with the cloud core.

\section{DISCUSSION}

The outcome of these simulations and the results given here are a first order proof of principle. From the current scenario it seems possible that every supernova with a progenitor of $20-40 \mathrm{M}_{\odot}$ can produce a system with solarlike ${ }^{26} \mathrm{Al}$ abundances by triggering a nearby $(\approx 5 \mathrm{pc})$ cloud core into collapse. However, we only tested a small range of the parameter space. Future simulations are desirable to further probe the likelihood. A first issue would be that we assume the (idealized) analytical Sedov-Solution for the blast wave. A more realistic treatment for the entire evolution of the shock from the progenitor is required in the future. This is of course computationally very expensive. Nevertheless, it is important to test e.g. the fragmentation and cooling of the shock front during the $8 \mathrm{kyr}$ before it hits the cloud core. Another area to improve would be to include more detailed effects. For example, the core could be initially rotating. We assume here that the high shock velocities 


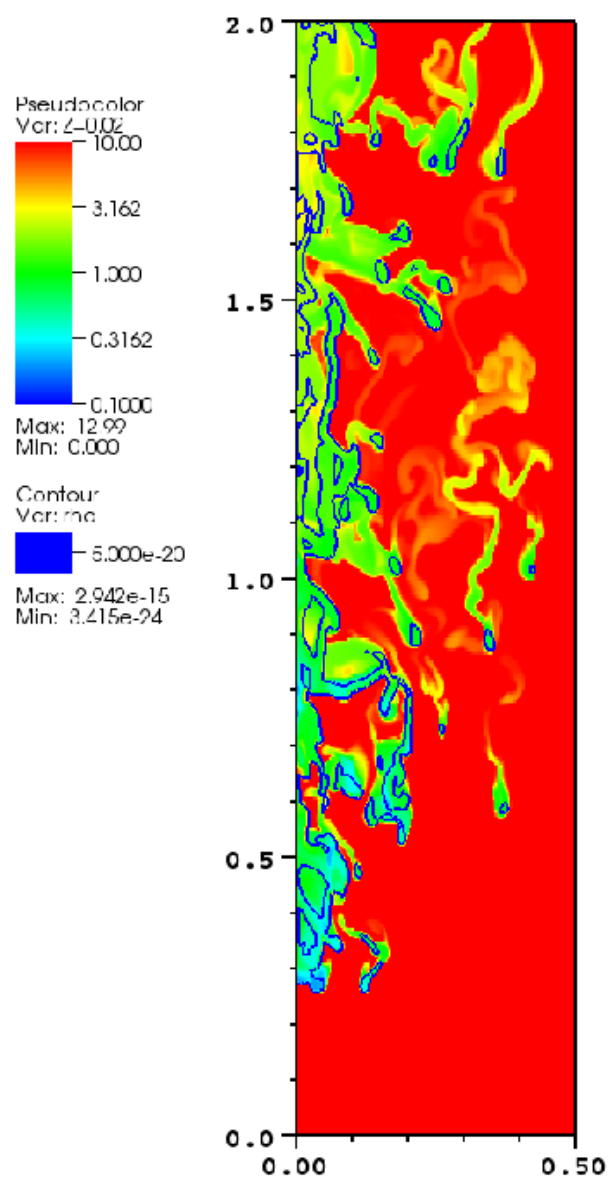

Fig. 5. - The tracer field, which follows the supernova gas at $t=$ $8.33 \mathrm{kyr}$. Color coded is the ratio ${ }^{26} \mathrm{Al} /{ }^{27} \mathrm{Al}$, the required value as inferred from CAI measurements is unity (green). The length scale is given in units of the radius of the initial cold core $\left(R_{0}=0.21 \mathrm{pc}\right)$. The color coding here is for a $40 \mathrm{M} \odot$ progenitor at 5 pc distance and the supernova gas getting fully mixed within the entire supernova bubble. The contours show the density distribution. As it can be seen the collapsing pre-solar gas gets sufficiently enriched in this case.

dominate over the rotation, but this should be further investigated. Furthermore, we neglect magnetic fields. They are of course present and should be involved in various processes, e.g. the details of the shock front and especially later on in the disk formation and the removement of angular momentum. In the early stages, however, the kinetic energy of the shock plays the dominant role. Still, it is worthwhile to investigate this regime of the parameter space in future simulations.

A remaining issue is the production of heavy SLRs like ${ }^{53} \mathrm{Mn}$ and ${ }^{60} \mathrm{Fe}$. Gounelle et al. (2009) suggest that the observed amount of ${ }^{60} \mathrm{Fe}$ can be inherited from the ISM. Still, the amount of galactic ${ }^{26} \mathrm{Al}$ is an order of magnitude lower than in the Solar System (Diehl 2006). Thus, a different enrichment mechanism for ${ }^{26} \mathrm{Al}$ is still needed. In addition, in the supernova-enrichment scenario, ${ }^{60} \mathrm{Fe}$ and especially ${ }^{53} \mathrm{Mn}$ are over-produced by a factor of 10-100 relative to their abundance in meteorites when compared to the lighter SLRs ${ }^{41} \mathrm{Ca}$ and ${ }^{26} \mathrm{Al}$ (e.g. Rauscher et al. 2002; Gaidos et al. 2009). This is often used as an argument against the supernova-

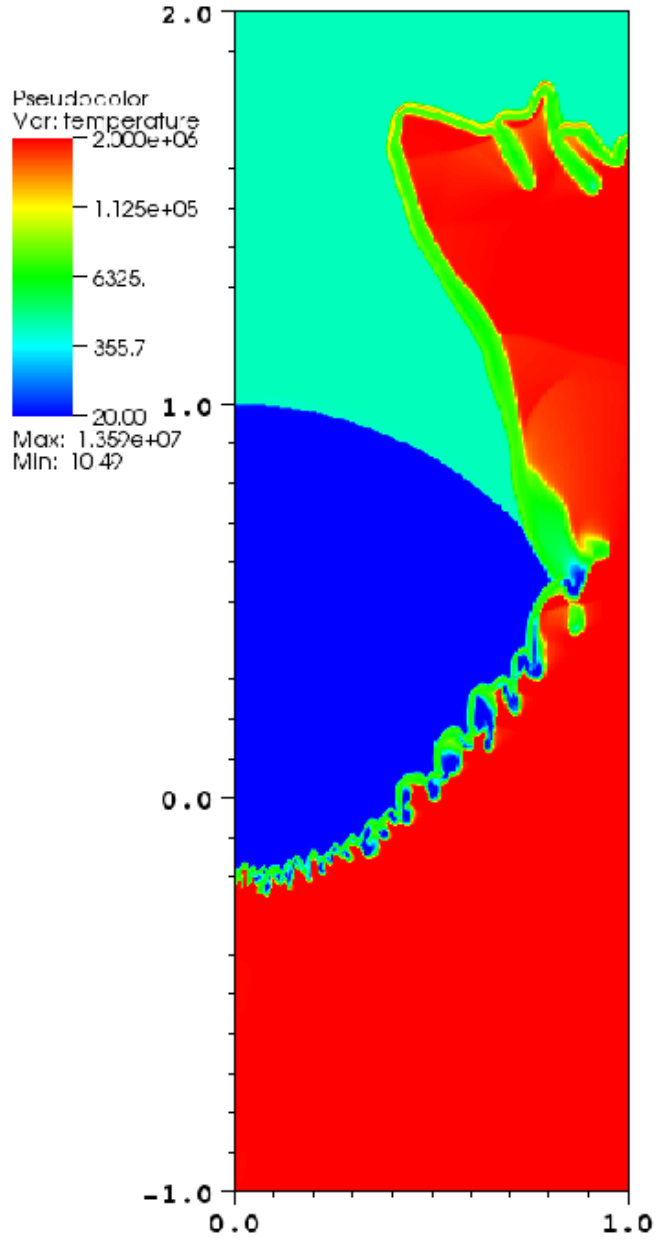

FIG. 6.- The temperature distribution at a much earlier stage $(t=1.25 \mathrm{kyr})$. Blue is cold, red is hot. The length scale is given in units of the radius of the initial cold core $\left(R_{0}=0.21 \mathrm{pc}\right)$. As can be seen, the enriched gas cools behind/within the shock-front and only later gets mixed with the pre-existing cold gas. The mixing happens between the two cold phases, rendering it more efficient.

enrichment scenario. However, there are several possible solutions. First of all, the precise determination of abundances, especially of radioactive isotopes in supernovae remains challenging. Second, there are alternative models, e.g. fallback-mixing within supernovae. Here, the innermost layers (commonly within the Si-burning layer) fall back onto the star. Therefore, there are much less SLRs heavier than Si within the supernova shock (e.g. Takigawa et al. 2008). Third, as it is an overproduction, not a lack of these SLRs, there could be different incorporation efficiencies into CAIs and meteorites, which are not yet well understood.

On a side note, the distribution of the supernova yield in the entire bubble versus the distribution of it in an outer rim gives different results (cf Table 2). As the iron is produced in layers further inside it is valid to speculate that the different ratios might be due to an homogeneous distribution of ${ }^{60} \mathrm{Fe}$ in the entire bubble, whereas ${ }^{26} \mathrm{Al}$ might be concentrated in an outer layer. This scenario is of course only valid if the initial distribution of elements in the supernova is conserved while the bubble expands 


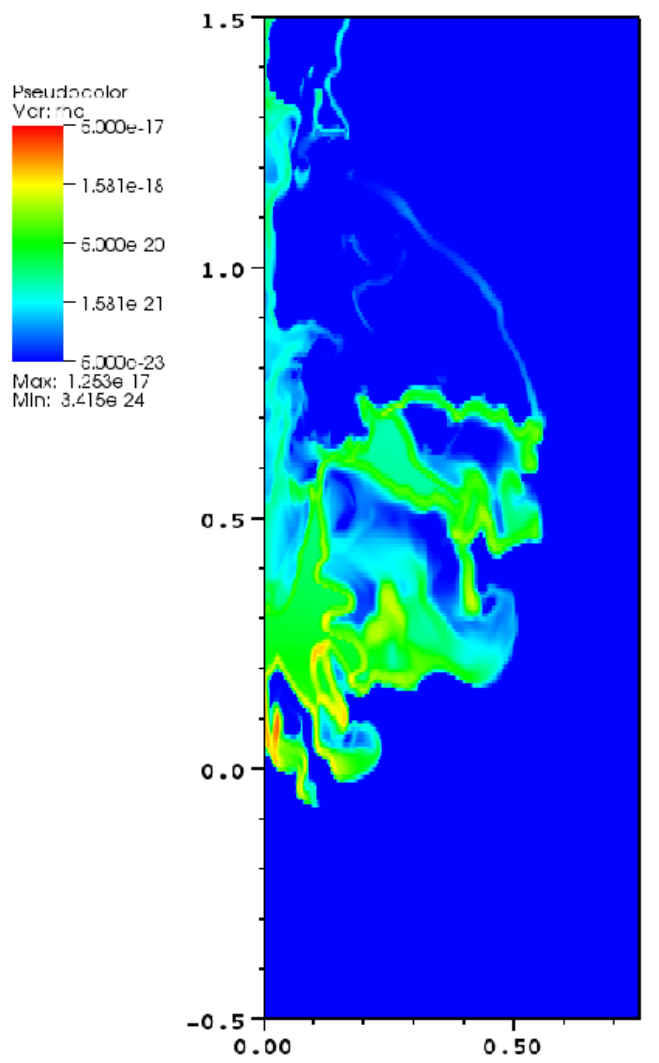

FIG. 7.- Zoom in into the final stage of Case II at $t=14.66 \mathrm{kyr}$. Color coded is the density, the length scale is given in units of the radius of the initial cold core $\left(R_{0}=0.21 \mathrm{pc}\right)$. Not even the densest regions undergo gravitational collapse.

to $5 \mathrm{pc}$.

The different abundances of SLRs are often used to calibrate the delay between the supernova and the formation of CAIs. Mostly, these calculations give values of about a Myr (Takigawa et al. 2008: $0.7 \mathrm{Myr}$, Looney et al. 2006: 1.8 Myr). This contradicts the fast formation time required to produce the small spread observed in the CAIs. In our opinion, the supernova yields of SLRs are at present not understood well enough to derive meaningful delay time scales between supernova and CAI formation. Especially, since there might be different processes during the disk formation, which could also explain the different abundances of SLRs.

In our simulations we find that a distance $D \approx 5 \mathrm{pc}$ is optimal to keep the balance of survivability for the preexisting cold clump and the sufficient enrichment. This is larger than previous estimates - e.g. Looney et al. (2006) derive a distance smaller than $4 \mathrm{pc}$ from analytical estimates. The reason for this is the efficient mixing as discussed in 3.2 .2 (see also Fig. 6).

For the survivability of the pre-solar clump we assume a quite high density and short lifetime for the HII-region. This leads to a small size, i.e. a small distance for the pre-existing clump from the $\mathrm{O}$-star. The general picture would be the ionization front running into the paternal filament, from which the O-star originally formed. On a related note, this leads to the assumed high density of the ambient surrounding of $n=100 \mathrm{~cm}^{-3}$ (accord-

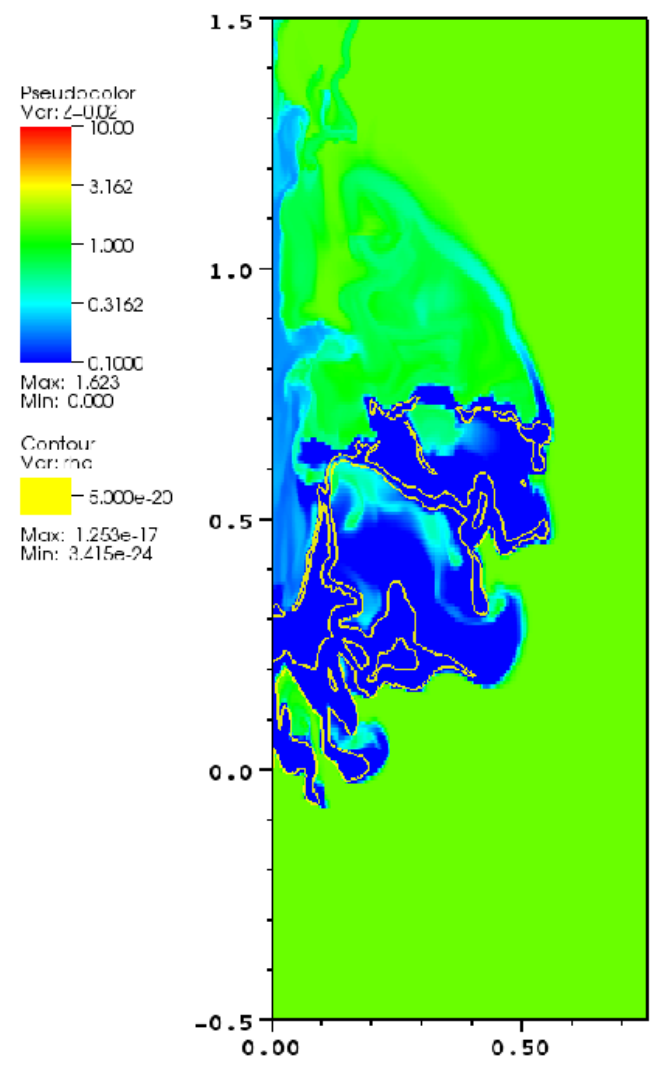

FIG. 8.- The tracer field, which follows the supernova gas for case II at $t=14.66 \mathrm{kyr}$. Color coded is the ratio ${ }^{26} \mathrm{Al} /{ }^{27} \mathrm{Al}$, the required value as inferred from CAI measurements is unity (green). The length scale is given in units of the radius of the initial cold core $\left(R_{0}=0.21 \mathrm{pc}\right)$. The color coding here is for a $40 \mathrm{M}_{\odot}$ progenitor at $10 \mathrm{pc}$ distance and the supernova gas getting fully mixed within the entire supernova bubble. The contours show the density distribution. The only regions where the cold gas gets slightly enriched are the colliding flanks in the back. Furthermore, no region undergoes gravitational collapse.

ing to the analytical evolution of an HII-region, see e.g. Shu 1991). This value is well within the observed range. Lefloch et al. (2002) estimate the density in the Trifid Nebula to be $50 \mathrm{~cm}^{-3}$ whereas Rubin et al. (2011) find values of $87-714 \mathrm{~cm}^{-3}$ and $74-1041 \mathrm{~cm}^{-3}$ for different lines in optical and infrared observations, respectively. In turn, the chosen value leads to a high density inside the supernova bubble, i.e. a very strong dilution of the enriched material. Thus, optimistic conditions for the survival lead to very conservative or pessimistic assumptions for the dilution. In addition, $\mathrm{O} / \mathrm{B}$-stars tend to form in bigger associations, therefore there would be more ionizing flux than from a single O-star. Furthermore, we neglect the effect of stellar winds. Altogether, the conditions for the survivability have been chosen quite optimistically to have a cold core close enough to the O-star to be sufficiently enriched $(D \approx 5 \mathrm{pc})$. Still, as the observations around various HII-regions show, pillar likestructures and therefore cold clumps are observed frequently at these distances.

On a more technical side, these simulations are still only in $2 \mathrm{D}$, so some physical processes might not be well resolved. This might apply to the gravitational collapse of two fronts colliding. However, colliding fronts of 
these speeds and densities have been shown to become gravitationally unstable with full 3D simulations (e.g. Gritschneder et al. 2009a). Another short-coming of 2D simulations might be the different behavior of instabilities and mixing in 2D and 3D (e.g. Stone \& Gardiner 2007). This should be less of an issue here, as the mixing appears in the cold phase and is not solely due to hydrodynamical instabilities, which would be less well represented in 2D (see Fig. 6). The mixing is mainly influenced by the cooling, which can be well approximated in 2D. Nevertheless, further simulations in full 3D would be desirable to address this issues.

\section{CONCLUSIONS \& OUTLOOK}

In this study, we focus on the hydrodynamical interaction of a supernova with the pre-solar cloud core. A realistic supernova shock wave is assumed to interact with the cloud. This leads to the conclusion that it is possible to enrich a pre-existing clump sufficiently with ${ }^{26} \mathrm{Al}$ to explain the observed ratio in the Solar System. In order to survive the previous HII-region and still be close enough to be sufficiently enriched and triggered into collapse, it turns out that $D \approx 5 \mathrm{pc}$ is an ideal distance.

The previously stable core gets enriched and is triggered into gravitational collapse within $t \approx 8 \mathrm{kyr}$ after the first interaction with the supernova shock-wave. The further collapse until the formation of the CAIs, which takes places at temperatures below $1800 \mathrm{~K}$, can be estimated to be shorter than $\simeq 10 \mathrm{kyr}$. Whether the collapse finally goes all the way down to the condensation of the CAIs within $18 \mathrm{kyr}$ or if there is an intermediate stage with a hot disk where the pre-meteorite gas is well mixed remains to be investigated. In either case, the CAIs will be able to grow fast enough to the observed $\mathrm{cm}$-sizes within the background density of a disk, as shown in Appendix A. The hot disk, however, can as well only be achieved by a very violent (i.e. supernova triggered) collapse. In addition, the subsequent expansion and cooling of the disk will be rapid at these high densities so it is preferable if the material is well mixed before.

On a side note, it is very interesting that the mixing occurs mainly by material from the supernova shock, which already cooled. This cooled portion interacts with the pre-existing cold gas in the surrounding of the progenitor star, whereas most of the supernova enriched material stays in the hot component. Therefore, it might be possible to determine the efficiency of enrichment by supernova feedback in the much larger context of galaxies by investigating the cooling rates within supernova shocks.

Altogether, the precise time and location of the grain formation has to be determined by future investigations. In this work, we prove that it is possible to trigger a pre-existing stable core into collapse by a very nearby supernova $(D \approx 5 \mathrm{pc})$. In addition, we show for the first time that the collapsing core can be enriched sufficiently with SLRs to explain the observed abundances, even if the enriched material is not assumed to be in the shock initially, but instead distributed homogeneously within the entire supernova bubble.

We would like to thank the referees for valuable comments on the manuscript. We thank Josh Wimpenny for carefully proof reading the final manuscript. M.G. acknowledges funding by the China National Postdoc Fund Grant No. 20100470108 and the National Science Foundation of China Grant No. 11003001. D.N.C.L. acknowledges support by NASA grant NNX07AI88G, NNX08AL41G and NNX08AM84G as well as the NSF grant AST-0908807. Q.Z.Y. acknowledges NASA Cosmochemistry grant NNX08AG57G and Origins of Solar Systems grant NNX09AC93G. The work by S.D.M. was performed under the auspices of the Lawrence Livermore National Security, LLC under contract No. DE-AC5207NA27344.

\section{REFERENCES}

Adams, F. C. 2010, ARA\&A, 48, 47

Amelin, Y., Krot, A. N., Hutcheon, I. D., \& Ulyanov, A. A. 2002, Science, 297, 1678

Anninos, P., Fragile, P. C., \& Murray, S. D. 2003, ApJS, 147, 177

Arnould, M., Paulus, G., \& Meynet, G. 1997, A\&A, 321, 452

Arthur, S. J., Henney, W. J., Mellema, G., De Colle, F., \& VázquezSemadeni, E. 2011, ArXiv e-prints

Boehringer, H., \& Hensler, G. 1989, A\&A, 215, 147

Bonnor, W. B. 1956, MNRAS, 116, 351

Boss, A. P. 2011, ApJ, 739, 61

Boss, A. P., Ipatov, S. I., Keiser, S. A., Myhill, E. A., \& Vanhala, H. A. T. 2008, ApJ, 686, L119

Boss, A. P., \& Keiser, S. A. 2010, ApJ, 717, L1

Boss, A. P., Keiser, S. A., Ipatov, S. I., Myhill, E. A., \& Vanhala, H. A. T. 2010, ApJ, 708, 1268

Cameron, A. G. W., Hoeflich, P., Myers, P. C., \& Clayton, D. D. 1995, ApJ, 447, L53+

Cameron, A. G. W., \& Truran, J. W. 1977, icarus, 30, 447

Dalgarno, A., \& McCray, R. A. 1972, ARA\&A, 10, 375

Dauphas, N., \& Chaussidon, M. 2011, Annual Review of Earth and Planetary Sciences, 39, 351

Desch, S. J., Connolly, Jr., H. C., \& Srinivasan, G. 2004, ApJ, 602, 528

Diehl, R. 2006, New Astronomy Reviews, 50, 534

Duprat, J., \& Tatischeff, V. 2007, ApJ, 671, L69

-. 2008, New Astronomy Reviews, 52, 463

Gaidos, E., Krot, A. N., Williams, J. P., \& Raymond, S. N. 2009, ApJ, 696, 1854

Garaud, P., \& Lin, D. N. C. 2007, ApJ, 654, 606
Goldsmith, P. F., Heyer, M., Narayanan, G., Snell, R., Li, D., \& Brunt, C. 2008, ApJ, 680, 428

Gounelle, M., \& Meibom, A. 2008, ApJ, 680, 781

Gounelle, M., Meibom, A., Hennebelle, P., \& Inutsuka, S.-i. 2009, ApJ, 694, L1

Gritschneder, M., Burkert, A., Naab, T., \& Walch, S. 2010, ApJ, 723,971

Gritschneder, M., Naab, T., Burkert, A., Walch, S., Heitsch, F., \& Wetzstein, M. 2009a, MNRAS, 393, 21

Gritschneder, M., Naab, T., Walch, S., Burkert, A., \& Heitsch, F. 2009b, ApJ, 694, L26

Grossman, L. 2002, Meteoritics and Planetary Science Supplement, 37,57

Heitsch, F., Hartmann, L. W., \& Burkert, A. 2008, ApJ, 683, 786

Herwig, F. 2005, ARA\&A, 43, 435

Hester, J. J. et al. 1996, AJ, 111, 2349

Hsu, W., Wasserburg, G. J., \& Huss, G. R. 2000, Earth and Planetary Science Letters, 182, 15

Hurley, J. R., Pols, O. R., \& Tout, C. A. 2000, MNRAS, 315, 543

Huss, G. R., Meyer, B. S., Srinivasan, G., Goswami, J. N., \& Sahijpal, S. 2009, Geochim. Cosmochim. Acta, 73, 4922

Ida, S., \& Lin, D. N. C. 2004, ApJ, 604, 388

Jacobsen, B., Yin, Q., Moynier, F., Amelin, Y., Krot, A. N. Nagashima, K., Hutcheon, I. D., \& Palme, H. 2008, Earth and Planetary Science Letters, 272, 353

Kastner, J. H., \& Myers, P. C. 1994, ApJ, 421, 605

Krumholz, M. R., Stone, J. M., \& Gardiner, T. A. 2007, ApJ, 671, 518 
Lee, T., Papanastassiou, D. A., \& Wasserburg, G. J. 1976, Geophys. Res. Lett., 3, 41

Lefloch, B., Cernicharo, J., Rodríguez, L. F., Miville-Deschênes, M. A., Cesarsky, D., \& Heras, A. 2002, ApJ, 581, 335

Lodders, K. 2003, ApJ, 591, 1220

Looney, L. W., Tobin, J. J., \& Fields, B. D. 2006, ApJ, 652, 1755

MacPherson, G. J., Bullock, E. S., Janney, P. E., Davis, A. M. Wadhwa, M., \& Krot, A. N. 2007, in Lunar and Planetary Institute Science Conference Abstracts, Vol. 38, Lunar and Planetary Institute Science Conference Abstracts, 1378-+

MacPherson, G. J., Davis, A. M., \& Zinner, E. K. 1995, Meteoritics, 30, 365

Marhas, K. K., Goswami, J. N., \& Davis, A. M. 2002, Science, 298, 2182

McCaughrean, M. J., \& Andersen, M. 2002, A\&A, 389, 513

McKeegan, K. D., Chaussidon, M., \& Robert, F. 2000, Science, 289,1334

Mellema, G., Arthur, S. J., Henney, W. J., Iliev, I. T., \& Shapiro, P. R. 2006, ApJ, 647, 397

Myers, P. C. 2009, ApJ, 700, 1609

Nath, B. B., Laskar, T., \& Shull, J. M. 2008, ApJ, 682, 1055

Nomoto, K., Tominaga, N., Umeda, H., Kobayashi, C., \& Maeda, K. 2006, Nuclear Physics A, 777, 424

Ouellette, N., Desch, S. J., Bizzarro, M., Boss, A. P., Ciesla, F., \& Meyer, B. 2009, Geochim. Cosmochim. Acta, 73, 4946

Ouellette, N., Desch, S. J., \& Hester, J. J. 2010, ApJ, 711, 597

Ouellette, N., Desch, S. J., Hester, J. J., \& Leshin, L. A. 2005, in Astronomical Society of the Pacific Conference Series, Vol. 341, Chondrites and the Protoplanetary Disk, ed. A. N. Krot, E. R. D. Scott, \& B. Reipurth, 527-+

Rauscher, T., Heger, A., Hoffman, R. D., \& Woosley, S. E. 2002, ApJ, 576, 323

Rubin, R. H., Simpson, J. P., O’Dell, C. R., McNabb, I. A., Colgan, S. W. J., Zhuge, S. Y., Ferland, G. J., \& Hidalgo, S. A. 2011, MNRAS, 410, 1320

Ruden, S. P., \& Lin, D. N. C. 1986, ApJ, 308, 883

Sedov, L. I. 1959, Similarity and Dimensional Methods in Mechanics (Academic Press)
Shu, C.-W., \& Osher, S. 1988, Journal of Computational Physics, 77,439

Shu, F. 1991, Physics of Astrophysics, Vol. II: Gas Dynamics (University Science Books)

Shu, F. H., Shang, H., Glassgold, A. E., \& Lee, T. 1997, Science, 277,1475

Shu, F. H., Shang, H., Gounelle, M., Glassgold, A. E., \& Lee, T. 2001, ApJ, 548, 1029

Stone, J. M., \& Gardiner, T. 2007, ApJ, 671, 1726

Strömgren, B. 1939, ApJ, 89, 526

Takigawa, A., Miki, J., Tachibana, S., Huss, G. R., Tominaga, N., Umeda, H., \& Nomoto, K. 2008, ApJ, 688, 1382

Tatischeff, V., Duprat, J., \& de Séréville, N. 2010, ApJ, 714, L26

Taylor, G. 1950, Royal Society of London Proceedings Series A, 201, 159

Thrane, K., Bizzarro, M., \& Baker, J. A. 2006, ApJ, 646, L159

Trigo-Rodríguez, J. M., García-Hernández, D. A., Lugaro, M., Karakas, A. I., van Raai, M., García Lario, P., \& Manchado, A. 2009, Meteoritics and Planetary Science, 44, 627

Villeneuve, J., Chaussidon, M., \& Libourel, G. 2009, Science, 325, 985

Walch, S., Burkert, A., Whitworth, A., Naab, T., \& Gritschneder, M. 2009, MNRAS, 400, 13

Walch, S., Naab, T., Whitworth, A., Burkert, A., \& Gritschneder, M. 2010, MNRAS, 402, 2253

Wasserburg, G. J., Gallino, R., Busso, M., Goswami, J. N., \& Raiteri, C. M. 1995, ApJ, 440, L101

Whitworth, A. P., Bhattal, A. S., Chapman, S. J., Disney, M. J., \& Turner, J. A. 1994, A\&A, 290, 421

Williams, J. P., \& Gaidos, E. 2007, ApJ, 663, L33

Woosley, S. E., \& Weaver, T. A. 1995, ApJS, 101, 181

Young, E. D., Simon, J. I., Galy, A., Russell, S. S., Tonui, E., \& Lovera, O. 2005, Science, 308, 223

\section{ASSEMBLY AND GROWTH OF CAIS}

Assuming that Aluminum sticks perfectly to the grains, the growth rate $\dot{M}$ for a particle of size $a$ at a certain radius within the disk with a relative velocity $v_{\text {rel }}$ is

$$
\dot{M}=\pi a^{2} \rho_{m} v_{\text {rel }},
$$

where $\rho_{\mathrm{m}}$ is the background density of the CAI-forming material, e.g. ${ }^{27} \mathrm{Al}$. If we assume that, as the particle grows the density of the particle $\rho_{\mathrm{p}}$ as well as $\rho_{\mathrm{m}}$ remain constant, we can write

$$
4 \pi a^{2} \dot{a} \rho_{\mathrm{p}}=\pi a^{2} \rho_{\mathrm{m}} v_{\mathrm{rel}}
$$

and solving for $\dot{a}$

$$
\dot{a}=\frac{\rho_{\mathrm{m}}}{4 \rho_{\mathrm{p}}} v_{\mathrm{rel}} .
$$

If we ignore the migration of particle, that is, the particle stays in a certain radius $r$ as it grows, the radius $a$ simply grows linearly with time

$$
a=a_{0}+\frac{\rho_{\mathrm{m}}}{4 \rho_{\mathrm{p}}} v_{\mathrm{rel}} t=a_{0}+k t,
$$

where the growth rate $k=\frac{\rho_{\mathrm{m}}}{4 \rho_{\mathrm{p}}} v_{\text {rel }}$ is a function of radius. Since we investigate a longer period of time $(t \sim 20 \mathrm{kyr})$, the initial particle size is irrelevant, so we can assume $a_{0}=0$. Therefore,

$$
\frac{\rho_{\mathrm{m}}}{4 \rho_{\mathrm{p}}} v_{\mathrm{rel}}=\frac{a}{t}
$$

To assemble cm-size objects within $20 \mathrm{kyr}$, this gives:

$$
\frac{\rho_{\mathrm{m}}}{4 \rho_{\mathrm{p}}} v_{\text {rel }}=1.6 \times 10^{-17} \mathrm{~km} \mathrm{~s}^{-1} .
$$

Assuming a density $\rho_{\mathrm{p}}=1 \mathrm{~g} \mathrm{~cm}^{-3}$ and a relative velocity of $v_{\text {rel }}=1 \mathrm{~km} \mathrm{~s}^{-1}$, the resulting Aluminum background density has to be $\rho_{\mathrm{m}}=6.4 \times 10^{-17} \mathrm{~g} \mathrm{~cm}^{-3}$. With the total mass ${ }^{27} \mathrm{Al}$ of $M_{27} \mathrm{Al}=7.1 \times 10^{-5} \mathrm{M}_{\odot}$ (e.g. Lodders 2003) this leads to a required total background density of

$$
\rho_{\text {disk }}=9.0 \times 10^{-13} \mathrm{~g} \mathrm{~cm}^{-3},
$$


which is higher than the density in cloud cores, but can be easily reached in proto-stellar and proto-planetary disks.

On the one hand this is an optimistic scenario for the growth, since we assume a perfect sticking efficiency. On the other hand, the injected supernova material most likely already contains $\sim 0.1 \mu \mathrm{m}$-sized grains (e.g. Nath et al. 2008). Furthermore, we only take ${ }^{27} \mathrm{Al}$ into account here, whereas the CAIs are not formed by pure ${ }^{27} \mathrm{Al}$. 